\title{
Are antibiotics necessary during routine cystoscopic stent removal?
}

\author{
Joel E. Abbott ${ }^{1}$, Allison Han ${ }^{2}$, Michelle McDonald ${ }^{1}$, Charlie Lakin ${ }^{1}$, Roger L. Sur ${ }^{1}$ \\ ${ }^{1}$ Department of Urology, University of California, San Diego, San Diego, CA, USA; ${ }^{2}$ University of California San Diego School of Medicine, San \\ Diego, CA, USA \\ Contributions: (I) Conception and design: JE Abbott, M McDonald, RL Sur; (II) Administrative support: C Lakin, RL Sur; (III) Provision of study \\ materials or patients: JE Abbott, C Lakin, RL Sur; (IV) Collection and assembly of data: JE Abbott, A Han, M McDonald; (V) Data analysis and \\ interpretation: JE Abbott, A Han, M McDonald, RL Sur; (VI) Manuscript writing: All Authors; (VII) Final approval of manuscript: All Authors. \\ Correspondence to: Joel E. Abbott, DO; Roger L. Sur, MD. Department of Urology, University of California San Diego, 200 West Arbor Drive \#8897, \\ San Diego, CA 92103, USA. Email: jeabbott@ucsd.edu; rlsur@mail.ucsd.edu.
}

Background: The 2008 American Urological Association (AUA) Best Practice Statement on antimicrobial prophylaxis states that prophylaxis is not warranted for subjects with normal risk profile undergoing cystourethroscopy unless manipulation such as ureteral stent removal is performed. To date no studies have specifically assessed the need for antimicrobial prophylaxis during cystoscopic ureteral stent removal. We sought to determine the risk of infectious complications following cystoscopic stent removal with and without antimicrobial prophylaxis.

Methods: A retrospective review identified 70 subjects who underwent cystoscopic ureteral stent removal following kidney stone treatment, under the care of two separate urologists with differing practice patterns. Each cohort consisted of 35 subjects: with and without prophylactic antibiotics. Clinical variables assessed included demographics, type of stone intervention, prior urinary tract infection (UTI) history, immunocompromising comorbidities, antimicrobial class at time of stone intervention, and antimicrobial administration at cystoscopic stent removal. The primary outcome assessed was development of symptomatic UTI within 4 weeks after stent removal.

Results: Overall, 35 patients (50\%) received antimicrobial prophylaxis at the time of stent removal and $35(50 \%)$ did not receive antimicrobial prophylaxis, with no demographic or clinical differences between cohorts. Two patients in the antimicrobial cohort (6\%) developed a UTI and none of the patients who did not receive antimicrobial prophylaxis developed a UTI $(\mathrm{P}=0.15)$.

Conclusions: In our cohort study antimicrobial prophylaxis at the time of cystoscopic stent removal did not appear to provide a significant benefit in UTI prevention. Prospective studies would assist in validating these findings.

Keywords: Kidney calculi; kidney surgery; urinary tract infection (UTI); stents; urologic surgical procedures

Submitted Jul 05, 2016. Accepted for publication Jul 27, 2016.

doi: $10.21037 /$ tau.2016.08.13

View this article at: http://dx.doi.org/10.21037/tau.2016.08.13

\section{Introduction}

Healthcare associated infection is a major challenge to modern healthcare and patient safety. Infectious complications following urological procedures are a significant source of patient morbidity and mortality and consume healthcare resources; however widespread overuse of antibiotics has significantly contributed to the growing bacterial resistance. Balancing judicious antibiotics use to avoid iatrogenic microbial resistance against timely use of antibiotics to prevent morbidity of infectious complications continues to challenge health care providers. Reliance on literature to guide decision-making becomes critical for health care providers.

The introduction of the American Urological Association (AUA) Best Practice Statement has provided this necessary 
guidance to urologists across the variety of urological surgeries (1). Routine revisions to this statement have kept the recommendations contemporary and in keeping with evolving evidence. And yet not all recommendations have literature to support their statements, requiring generalizations to support some guidelines. In particular, the recommendation of antibiotic use during cystoscopic stent removal is based on this type of generalized data from transurethral resection literature findings. However, this recommendation seems to fail the intuitive test as a cystoscopic stent removal carries a much less invasive morbidity than either transurethral resection of prostate or bladder tumor. In this context, we hypothesize that antibiotics at time of cystoscopic stent removal provide minimal benefit. We therefore sought to determine if antibiotics indeed provided benefit in reducing infectious complications for cystoscopic stent removal procedure.

\section{Methods}

After Institutional Review Board was obtained (UCSD Human Research Protection Program \#160159), we retrospectively reviewed the medical records of all patients presenting to UC San Diego Health Comprehensive Kidney Stone Center clinic from October 2014 to October 2015 seeking to include any subject who underwent cystoscopic ureteral stent removal. A total of 70 subjects under the care of two separate urologists (Roger L. Sur \& Charlie Lakin) with differing practice patterns were identified in order to isolate two cohorts of subjects: 35 received (Roger L. Sur subjects) and 35 did not (Charlie Lakin subjects) receive prophylactic antibiotics. Cohort 1 utilized single dose antibiotics after the procedure, while cohort 2 did not utilize antibiotics after the procedure. As per the current AUA best practice policy statement the following antibiotics were utilized: fluoroquinolones, cephalosporins, aminoglycosides, or trimethoprim-sulfamethoxazole (TMP-SMX) (1).

\section{Inception cobort}

All subjects undergoing upper tract stone treatment, including percutaneous nephrolithotomy (PNL), ureteroscopy/lithotripsy (URS), or shock wave lithotripsy, had ureteral stents placed intraoperatively. All patients received $<24$ hours of prophylactic antibiotics at the time of their initial surgical procedure in accordance with our institution's protocol of weight-based dose of gentamycin and ampicillin. Vancomycin was used to replace ampicillin in patients with allergy concern and ceftriaxone was used to replace gentamycin for patients with impaired renal function glomerular filtration rate $(\mathrm{GFR})<60$. Patients presented 1-2 weeks post-operatively to have their stents removed in the clinic. Exclusion criteria included positive preoperative urine cultures, presence of indwelling external urinary drain tubes (Foley catheters, nephrostomy tubes), performance of clean intermittent catheterization, stent duration $>2$ weeks, and concurrent antimicrobial administration at time of cystoscopic stent removal.

\section{Primary outcome}

The primary outcome assessed was development of urinary tract infection (UTI) within 4 weeks after stent removal. UTI was defined in accordance with the standardized American College of Surgeons National Surgical Quality Improvement Program (ACS NSQIP) definition of postoperative UTI (2). ACS NSQIP standardized UTI definition is listed in Figure 1.

\section{Descriptive statistics}

Demographic variables were reported with mean, median and appropriate measures of variance for continuous variables and proportions for categorical variables. Other clinical variables reported included type of stone intervention, UTI history, immunocompromising comorbidities, antimicrobial class at time of stone intervention, and antimicrobial administration at cystoscopic stent removal.

\section{Inferential statistics}

Student's $t$-test was used to compare continuous variables as they were parametric in distribution. Pearson's chi-square/ Fisher's exact test was used for categorical data analysis and reported with odds ratio (OR) and $95 \%$ confidence intervals (CI). All $\mathrm{P}$ values were two-tailed and statistical significance was set at $\mathrm{P}<0.05$. Analyses were performed in SAS 9.3 (SAS Institute, Cary, NC, USA).

\section{Results}

A total of 122 medical charts were reviewed to obtain the 70 subjects meeting the inclusion criteria for the study: 35 (Roger L. Sur) subjects received prophylactic antibiotics at time of cystoscopic stent removal and 35 (Charlie Lakin) subjects did not received antibiotics at time of cystoscopic 


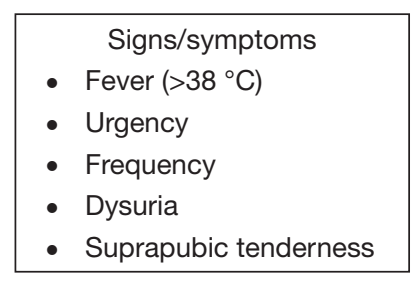

\section{Additional criteria}

- Dipstick test positive for leukocyte esterase and/or nitrate

- Pyuria (>10 WBCs/mm $/ \mathrm{mm}^{3}$ or $>3 \mathrm{WBC} / \mathrm{HPF}$ of unspun urine)

- Organisms on Gram stain of unspun urine

- Two urine cultures with repeated isolation of the same uropathogen with $>100 \mathrm{CFU} / \mathrm{mL}$ in non-voided specimen

- Urine culture with $<100,000 \mathrm{CFU} / \mathrm{mL}$ of single uropathogen in patient on appropriate antimicrobial therapy

- Physician's diagnosis

- Physician institutes antimicrobial therapy

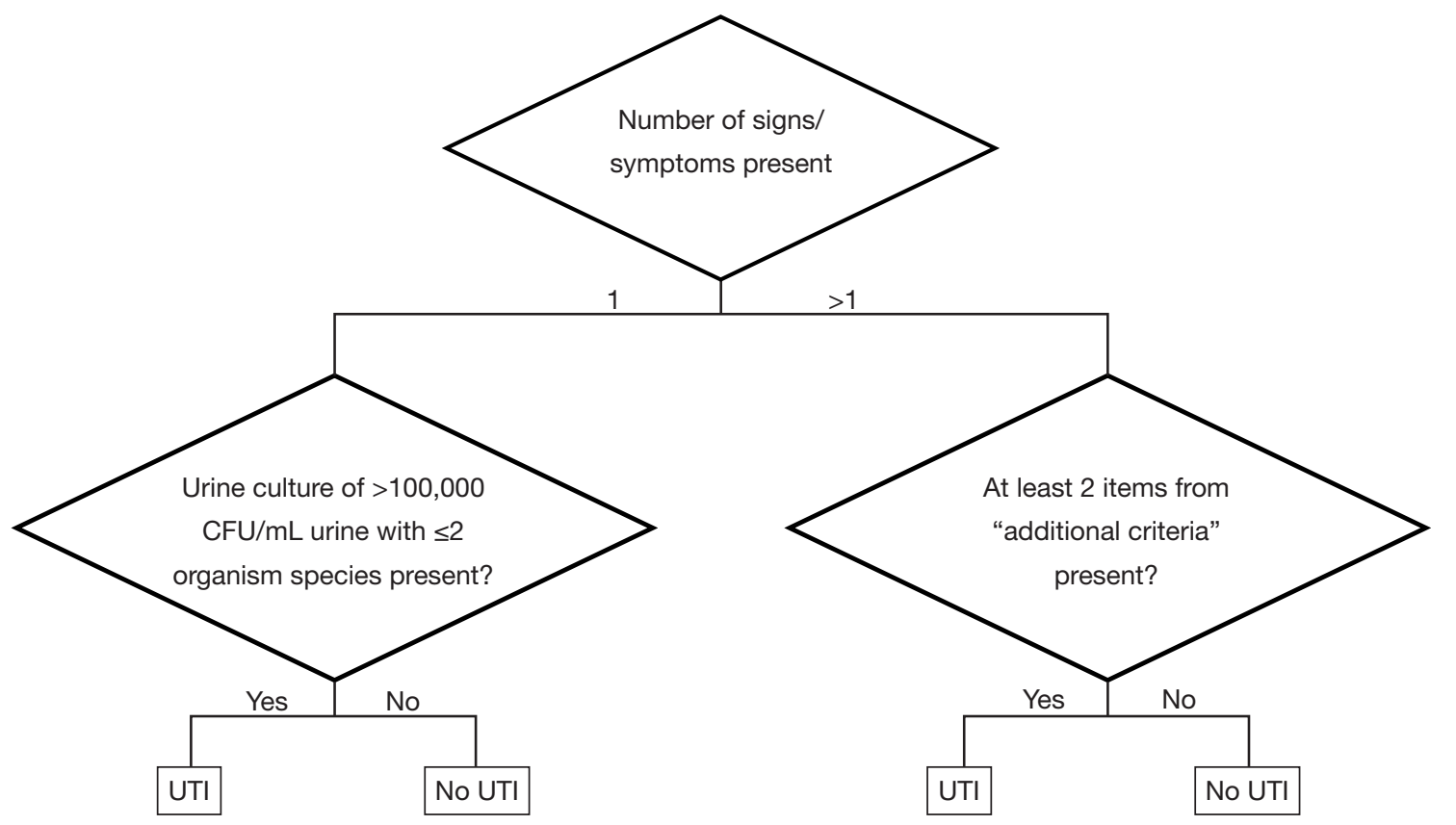

Figure 1 American College of Surgeons National Surgical Quality Improvement Program (ACS NSQIP) criteria to define post-operative UTI (2).

stent removal. No significant differences in either demographic or clinical variables were identified between these cohorts (Table 1), though cohort 1 had two subjects with human immunodeficiency virus (HIV) and one subject with history of chemotherapy $(\mathrm{P}=0.49$ and $\mathrm{P}=1.0$, respectively). Mean indwelling stent duration between the two groups was $9.09( \pm 3.23)$ days in cohort 1 and $9.94( \pm 2.99)$ days in cohort 2 . The antimicrobial-receiving cohort in this retrospective study received ciprofloxacin preferentially $(n=31,89 \%)$, gentamicin $(n=2,6 \%)$, cefalexin $(n=1,3 \%)$, and TMP-SMX ( $\mathrm{n}=1,3 \%)$. Antibiotic selection was based on physician preference influenced on patient allergy profile but in keeping with AUA Best Practice Statement.

With regard to the primary outcome, there was no statistical difference in UTI rates between the two groups $(\mathrm{P}=0.151)$. In the antimicrobial cohort, two patients $(6 \%)$ developed a UTI. In the cohort not receiving antimicrobial prophylaxis, none of the patients developed UTIs. Among the patients diagnosed with UTI, one UTI was secondary to $>100 \mathrm{k}$ colony-forming units (CFU) Staphylococcus epidermidis, while the other UTI was secondary to $>100 \mathrm{k}$ CFU Strepococci viridans. Both patients were treated to resolution with culture-specific oral antibiotics with immediate resolution of symptoms. Of note none of these subjects who suffered UTI's had history of HIV or chemotherapy.

The UTI secondary to Strepococci viridans occurred in a male who was HIV positive. The UTI secondary to Staphylococcus epidermdis occurred in a female with no comorbidities.

\section{Discussion}

The purpose of antimicrobial prophylaxis in surgical 
Table 1 Demographic and clinical variables of both cohorts

\begin{tabular}{lccc}
\hline Clinical variable & Cohort $1(\mathrm{abx})(\mathrm{n}=35)$ & Cohort 2 (no abx) $(\mathrm{n}=35)$ & $\mathrm{P}$ value (two-tailed) \\
\hline Gender, male & $20(57 \%)$ & $26(74 \%)$ & 0.21 \\
Age, mean & $57 \pm 14$ & $56 \pm 11$ & 0.86 \\
BMI, mean & $28 \pm 5.0$ & $29 \pm 6.2$ & 0.32 \\
Diabetes & $4(11 \%)$ & $8(23 \%)$ & 0.34 \\
HIV & $2(6 \%)$ & 0 & 0.49 \\
Chemotherapy & $1(3 \%)$ & 0 & 1.00 \\
Recurrent UTI & 7 & 4 & 0.51 \\
PNL & 3 & 6 & 0.48 \\
URS & 32 & 29 & 0.48 \\
Stent duration (days) & $9.09 \pm 3.23$ & $9.94 \pm 2.99$ & 0.25 \\
\hline
\end{tabular}

BMI, body mass index; HIV, human immunodeficiency virus; UTI, human immunodeficiency virus; PNL, human immunodeficiency virus; URS, ureteroscopy/lithotripsy.

procedures is not to sterilize the tissues but to reduce the colonization of microorganisms introduced at the time of operation to a level that the patient's immune system is able to overcome (3). Before an agent can be considered for use as a prophylactic antibiotic, there must be evidence that it reduces postoperative infection. Agents used must be safe, ideally economical and effective against organisms likely to be encountered in the surgical procedure. Postoperative UTIs are a major concern for morbidity in patients after urological procedures, and this concern is used to justify the use of prophylaxis with stent removal $(1,4)$.

However, we failed to find a benefit to antimicrobial prophylaxis within this cohort study. Perhaps the noninvasive nature to this procedure as well as paucity of risk factors explains the lack of significant differences in infectious complications. The difference between cystoscopy and cystoscopic stent removal is that a foreign body is grasped and removed from the urinary tract. Since this maneuver should reestablish the anti-refluxing mechanism of the ureteralvesicle junction (allowing continued passive renal drainage without ureteral reflux), bacteria within the bladder should not be transmitted retrograde, and colonized urine in the upper tract is permitted continuous drainage in an antegrade fashion. Obstruction is not routinely observed after simple stent removal following stone treatment (5). Furthermore, the ureteral stent functions as a surgical drain to the kidney.

The basis of the current antimicrobial prophylaxis during cystoscopic stent removal derives from AUA panel conclusion that similarities of these other cystoscopic procedures in terms of invasiveness and potential tissue trauma suggest that the data regarding transurethral resection of the prostate and bladder tumor reasonably can be extrapolated to other cystoscopic procedures with manipulation (1). The presumption that prior transurethral resection literature is translatable to this much less invasive procedure seems clinically irrational. Both are "invasive" but the similarities end there-as cystoscopic stent removal is no more invasive than Foley catheter removal. There is no tissue removal, no tissue trauma and really no "invasiveness" to stent removal from a clinical perspective. Given the lack of specific data to justify antibiotic use, juxtaposed with known increasing risks of antibiotic resistance, literature supporting prophylaxis should be ideally expected to enhance panels create recommendations (6).

However there may be specific risk factors that warrant prophylaxis at the time of stent removal-just as Foley catheter removal with risk factors merits antibiotics. Patients at risk for infectious complications following urologic intervention have previously been defined as patients with recent urosepsis, anomalies of the urinary tract, urinary obstruction, incomplete bladder emptying, chronic externalized urinary tract drains/catheters, and history of recurrent UTI's $(1,4,7)$. Until the literature provides better delineation of the at-risk populations the benefit to risk ratio tilts towards prophylaxis for this cohort.

The purpose of this retrospective analysis was to examine the efficacy and therefore necessity of providing antimicrobial prophylaxis at the time of cystoscopic stent removal with the primary end point of UTI incidence. By excluding patients who demonstrated positive preoperative urine cultures, had external drain tubes or catheterizations, concurrent antimicrobial administration, and stents for 
longer than 2 weeks, we attempted to eliminate possible confounding factors and assess uncomplicated patients with minimal baseline risk of UTI. We examined other variables that may generally increase risk for infection including immune-compromised state (cancer, diabetes mellitus, HIV), obesity, old age, smoking, though we did not exclude patients based upon these factors.

Our study has limitations, including its retrospective design and small sample size. Additionally, the small number of patients who developed UTIs may underpower the study, though the current $6 \%$ difference in this retrospective study would require prospective sample size of approximately 2,000 subjects. This retrospective study therefore provides the basis for future prospective trials to validate our findings. We acknowledge other limitations. The antibiotic regimen was not standardized and represented physician preference. It is conceivable that the prescribing urologist in cohort 1 treated higher risk patients with broader coverage antimicrobial.

\section{Conclusions}

Antibiotic prophylaxis at the time of cystoscopic stent removal following endoscopic stone treatment for simple stent removal did not decrease the risk of UTI in uncomplicated patients in this cohort study. This is a significant finding to explore, as unnecessary antimicrobial usage increases healthcare costs, places patients at risk for antimicrobial related adverse events, and potentially introduces resistant organisms to the community. Further prospective investigations should be considered to validate these findings.

\section{Acknowledgements}

None.

\section{Footnote}

Conflicts of Interest: The authors have no conflicts of interest

Cite this article as: Abbott JE, Han A, McDonald M, Lakin C, Sur RL. Are antibiotics necessary during routine cystoscopic stent removal? Transl Androl Urol 2016;5(5):784-788. doi: $10.21037 /$ tau.2016.08.13 to declare.

Ethical Statement: This study was done in accordance with UCSD IRB protocol \#160159. However, the study was considered exempt from IRB review as per federal regulations [45 CFR 46.101(b)]. The study met criteria for exempt category 4: research involving collection or study of existing data, documents, records, or specimens, the information is recorded by the researcher in such a manner that subjects cannot be identified, directly or through identifiers linked to the subjects.

\section{References}

1. Wolf JS Jr, Bennett CJ, Dmochowski RR, et al. Best practice policy statement on urologic surgery antimicrobial prophylaxis. J Urol 2008;179:1379-90.

2. National Surgical Quality Improvement Program: Noncardiac terms \& definitions, revisions to definitions 9-1-95. In: Managerial I Site Operations Manual St Louis, MO. St. Louis, MO: Continuing Education Center, 1995.

3. Mangram AJ, Horan TC, Pearson ML, et al. Guideline for Prevention of Surgical Site Infection, 1999. Centers for Disease Control and Prevention (CDC) Hospital Infection Control Practices Advisory Committee. Am J Infect Control 1999;27:97-132; quiz 133-4; discussion 96.

4. Matsumoto T, Kiyota H, Matsukawa M, et al. Japanese guidelines for prevention of perioperative infections in urological field. Int J Urol 2007;14:890-909.

5. Manger JP, Mendoza PJ, Babayan RK, et al. Use of renal ultrasound to detect hydronephrosis after ureteroscopy. J Endourol 2009;23:1399-402.

6. Patel N, Shi W, Liss M, et al. Multidrug resistant bacteriuria before percutaneous nephrolithotomy predicts for postoperative infectious complications. J Endourol 2015;29:531-6.

7. Grabe M, Bjerklund-Johansen TE, Botto H, et al. Guidelines on Urological Infections 2013. Available online: http://uroweb.org/guideline/urological-infections/ 